\title{
A feasibility study of a seismic survey along a mountain ridge using acoustic wave simulator
}

\author{
*Aoki, Naoshi ${ }^{1)}$, Mizohata, Shigeharu ${ }^{1)}$, Minegishi, Masato ${ }^{1)}$, \\ Nakajima, Yoshinari ${ }^{2)}$ and Asakawa, Eiichi ${ }^{3)}$ \\ 1) Japan Petroleum Exploration, Co.,Ltd, Tokyo, Japan. \\ 2) Japan National Oil Corporation, Tokyo, Japan.
}

3) JGI, Inc, Tokyo, Japan.

\begin{abstract}
Oil and gas explorations in mountainous regions are becoming more important to find unexplored possible reserves. Here, the seismic survey technique is a major exploration tool as well. However, difficulties often come from complicated topography change. For evaluating the topographic effects, we conducted the feasibility study for a 2D seismic survey in a particular mountainous area. In our study area, the locations where we can put sources or receivers are restricted, and it is possible to lay out dense sources and receivers on only one mountain road. Previously, reflection from mountainside or diffractions from topographic kink point were worried to damage reflection signals from subsurfaces. In this study, 3D acoustic wave simulation was conducted with the realistic topography model, and the calculated wavefield was examined. As the result, several different types of topography-related waves are identified. However, judging from this result and the other conditions, the seismic survey on a mountain ridge could be acceptable. And the acoustic simulator can be largely useful to the survey planning even though there were some limitations on the method.
\end{abstract}

\section{Introduction}

Oil and gas explorations in mountainous regions are becoming more important to find unexplored possible reserves. Here, the seismic survey technique is a major exploration tool as well. However, difficulties often come from complicated topography change. This feasibility study contains an evaluation of a $2 \mathrm{D}$ seismic survey in a particular mountainous area. The number of points, where we can put sources or receivers is restricted in the area, and the 2D survey along only one mountain road can be conducted with dense sources and receivers. However, earth's surface is the strongest reflector, so reflections from subsurfaces strongly reflect again on the mountainsides. The affection may be largest on a mountain ridge and damage the quality of the survey data. In such case, we might have to move the receiver line from the ridge to the foot of the mountain. On the other hand, kink points of the topography like ravine might generate diffraction energy, and then receiver line should be kept away from it. For evaluating these topographic affections onto the survey, 3D acoustic wave simulation with the realistic 
topography model was conducted.

\section{Methods}

In this study, we used the 3D acoustic seismic wave simulator, which adopted the pseudo-spectrum method (Sato 1997). The earth has an elastic nature; therefore, elastic waves should be considered ideally. However, computation of elastic waves requires a huge and impractical time, especially in three-dimensional cases. On the other hand, acoustic wave simulation can be achieved much faster than elastic wave simulation. And it seems enough just for evaluating topographic affection onto conventional seismic.

In addition, the pseudo-spectrum method has an advantage in computation time. The Fourier transform requires fewer grid points per wavelength than the finite difference method with equal accuracy. Even though we have to give a specific model that the number of grid must be exponential of 2, 3D full wave simulation can be carried out in a practical time. Such a quick and cheap method can be appropriate for many try and error approaches at the survey design.

\section{Model Building and Simulation}

The Geographical Survey Institute has published digital maps of Japan. The topographic data of $50 \mathrm{~m}$ meshes was used for the $1 \mathrm{st}$ interfaces of our velocity model (Figure 1). Below this interface, only one flat horizontal interface was incorporated. Then, $\mathrm{P}$ wave velocity was assigned to each layer.

Model and simulation parameters should be carefully selected because there is a trades-off between accuracy and calculation time (We evaluated them on a spreadsheet to stabilize the simulation result and save the computation time). Computation time for the method is, roughly, in proportion to $\mathrm{NX}^{*} \mathrm{NY} \mathrm{N}^{*} \mathrm{NZ} \mathrm{NT}^{*} \mathrm{NX} \sim \mathrm{NT}$ stand for number of grid in $\mathrm{X} \sim \mathrm{T}$ domain). If we try to describe the model precisely, the number of grids tends to increase and the calculation time will be unacceptably large even though the pseudo-spectrum method is used.

For example, if a velocity of $340 \mathrm{~m} / \mathrm{sec}$ is assigned to the air layer, the grid size should be less than $5 \mathrm{~m}$ to keep the maximum frequency at $30 \mathrm{~Hz}$. And then a number of cells is needed at least $512 * 512 * 512$ for the evaluation of the survey area. It takes 9 days for the simulation to this model on the Compaq Alpha server. Therefore, we decided to take an unrealistic parameter, such as air layer's velocity $=1000 \mathrm{~m} / \mathrm{s}$. Introduction of this artificial layer reduce the computation time to 7 hours.

The earth's surface position in the Z range was also carefully examined. All the source and receivers points are placed at the one half of grid cell depth from the 1 st interface. In the simulator, up-going energy reflects on the top of the model and creates a strong artifact. It might hide the topographic affections if the topography and the top of the model are too close. To avoid the interference within the time window of the reflection signal, the 1st interface was buried 
about one third of the $\mathrm{Z}$ range of the model. Figure 2 shows our final velocity model. The parameters of the velocity model and the simulation are shown in Table 1.

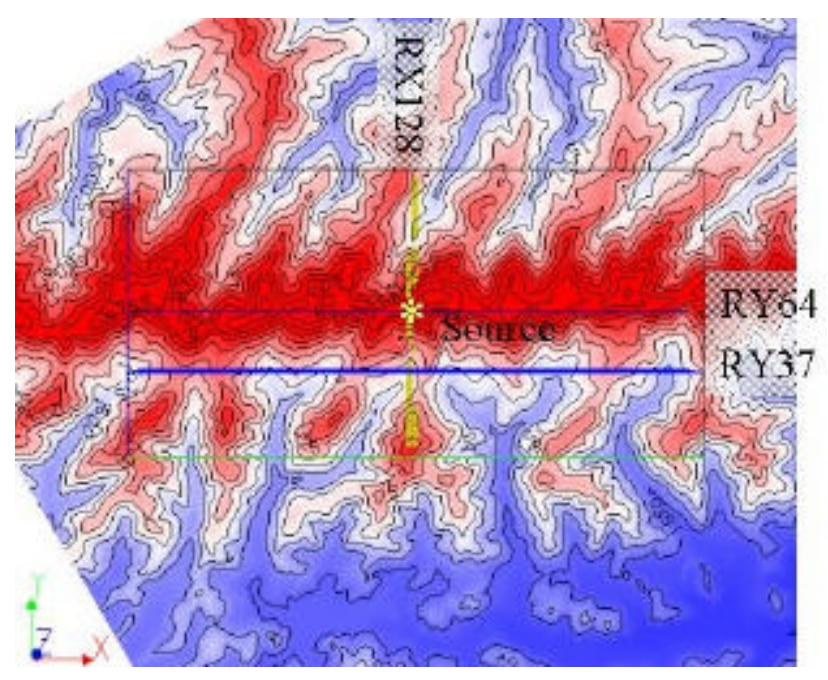

Figure 1: A 50m-meshed digital topographic map is used for the earth's surface model. The data are published by Geographical Survey Institute.

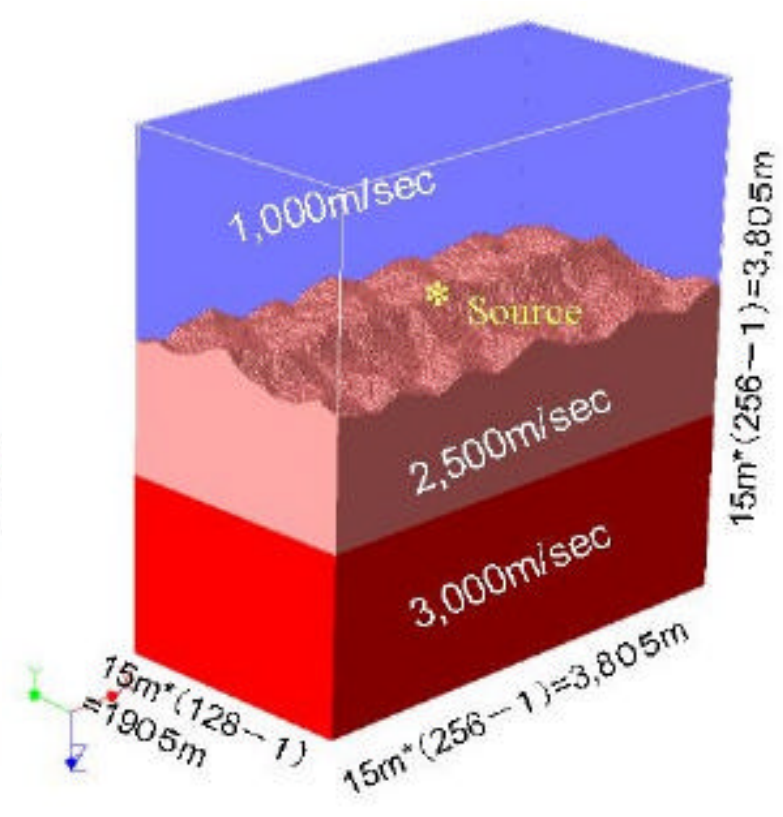

Figure 2: Velocity model has 1000, 2500 and $3000 \mathrm{~m} / \mathrm{sec}$ layer in order from the top. The earth's surface has the highest velocity contrast in this model. This model was created using GoCAD, which is an integrated computer application for 3D modeling, visualization, interpretation and geo-analysis.

Table 1: Parameters for the Velocity model and the Simulation

Velocity Model Parameters

Minimum velocity $(\mathrm{m} / \mathrm{s})^{*}$

$1000 \mathrm{~m} / \mathrm{s}$

Maximum velocity $(\mathrm{m} / \mathrm{s})^{*}$

$3000 \mathrm{~m} / \mathrm{s}$

Average velocity $(\mathrm{m} / \mathrm{s}) *$ (in rough)

$2500 \mathrm{~m} / \mathrm{s}$

Grid interval (m)

$15 \mathrm{~m}$

Number of Inline grids*

Number of Cross line grids* 
Number of Depth grids* 256

Inline length $3825 \mathrm{~m}$

Cross line length $1905 \mathrm{~m}$

Depth $3832 \mathrm{~m}$

Simulation parameters

Maximum Frequency of BP Zero phase wavelet $(\mathrm{Hz})^{*} \quad 30 \mathrm{~Hz}$

Minimum Frequency of BP Zero phase wavelet $(\mathrm{Hz}) \quad 6.7 \mathrm{~Hz}$

Sampling interval $1 \mathrm{msec}$

Recording time $\quad 3 \mathrm{sec}$

Estimated calculation time on the Alpha server $\quad 7$ hours/shot

The marked parameters by * were given to the spreadsheet for an evaluation. According to stability conditions, the other dependent parameters were automatically calculated or estimated.

\section{Simulation Results}

Source energy was set at the coordinate of $(128,64,97.5)$ that was the center of the area and one half of the grid interval below the 1st interface, which corresponded to the earth's surface. The simulation results are shown below. Figure 3 shows a series of the snapshots on RX128 (refer to Figure. 1). The waves, which propagated in the $1000 \mathrm{~m} / \mathrm{s}$ layer, are muted just for better visualization. Figure 4 shows the shot records on the RX128, RY64, and RY37. This calculation took about 7 hours by using 1 node of the Compaq Alpha Server. 


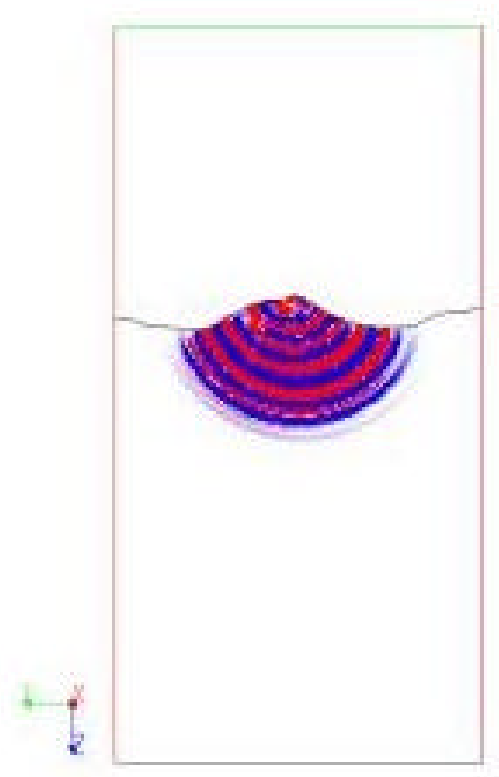

(a) $300 \mathrm{msec}$

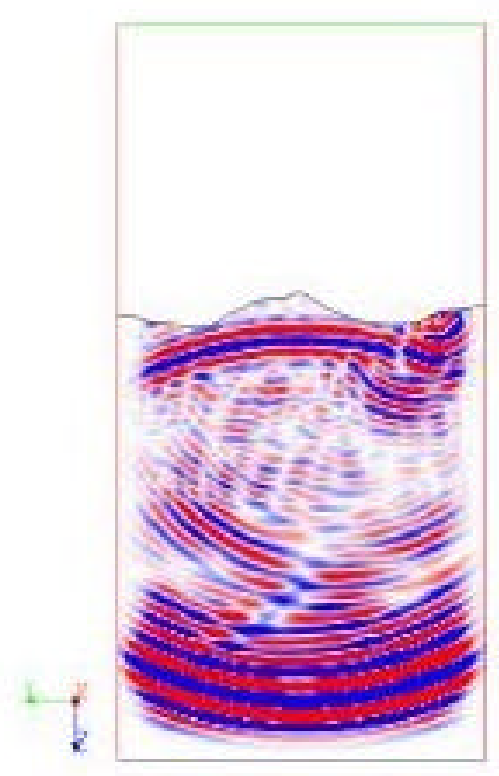

(d) $900 \mathrm{msec}$

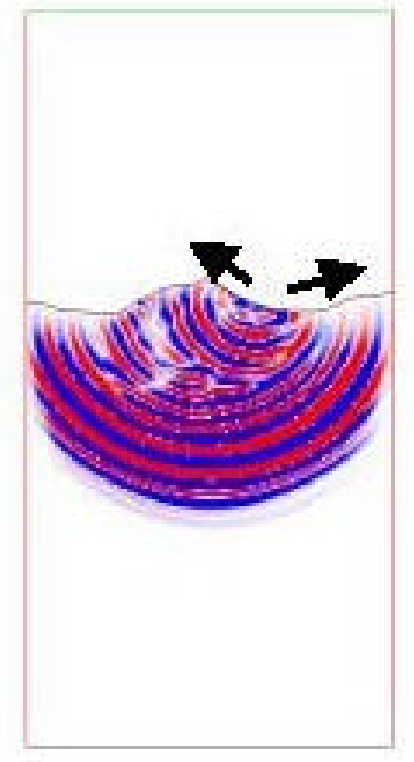

(b) $500 \mathrm{msec}$

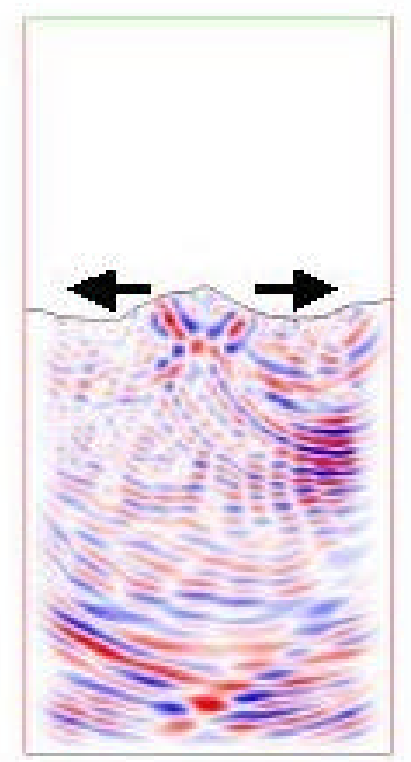

(e) $1100 \mathrm{msec}$

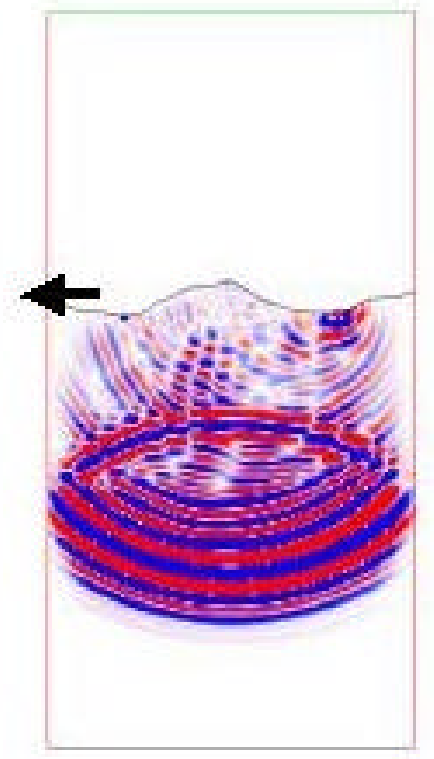

(c) $700 \mathrm{msec}$

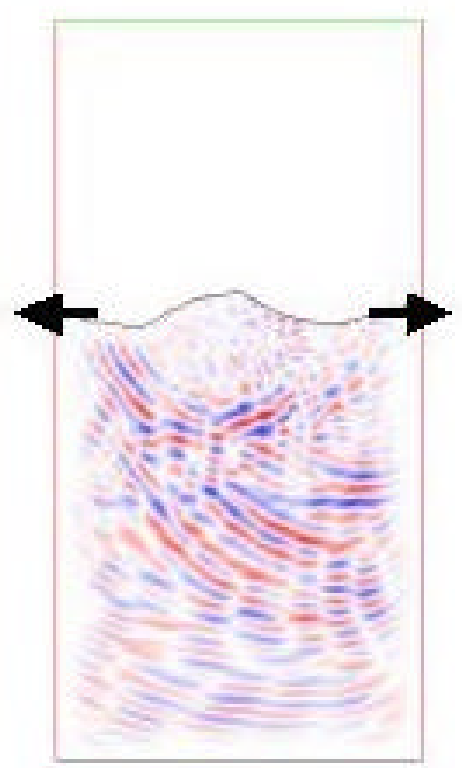

(f) $1300 \mathrm{msec}$

Figure 3: (a) $300 \mathrm{msec}$ after the shooting. Strong diffractions were generated at the topographic kink point on the right side of the mountain in (b) and (c). The arrows explain the apparent directions that the waves propagate along the surface. (d) shows reflection energy which propagates upward is going to contact with the earth's surface, and (e) and (f) show after the contact. In this series, we can see that the undulating topography causes energies that travel along the surface. 


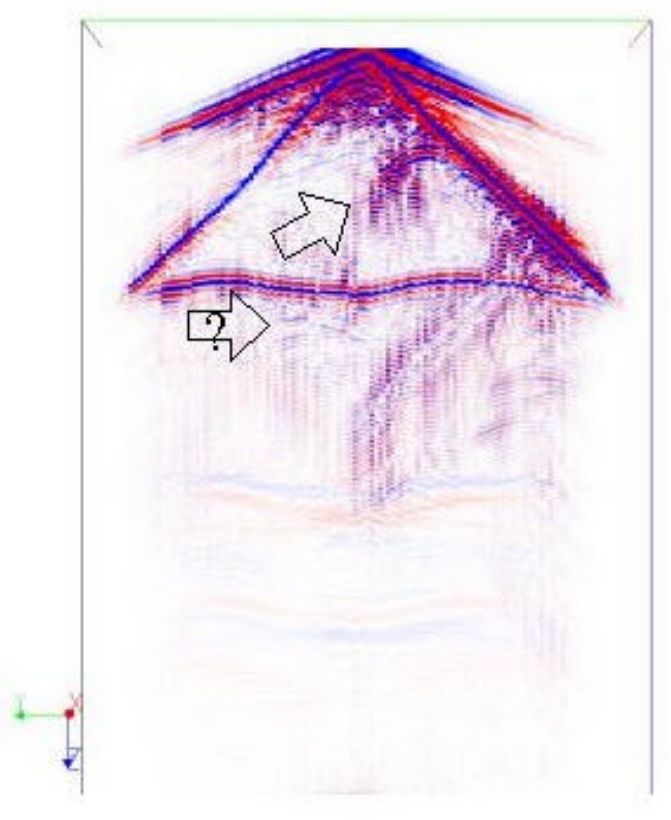

(a) $\mathrm{RX} 128$

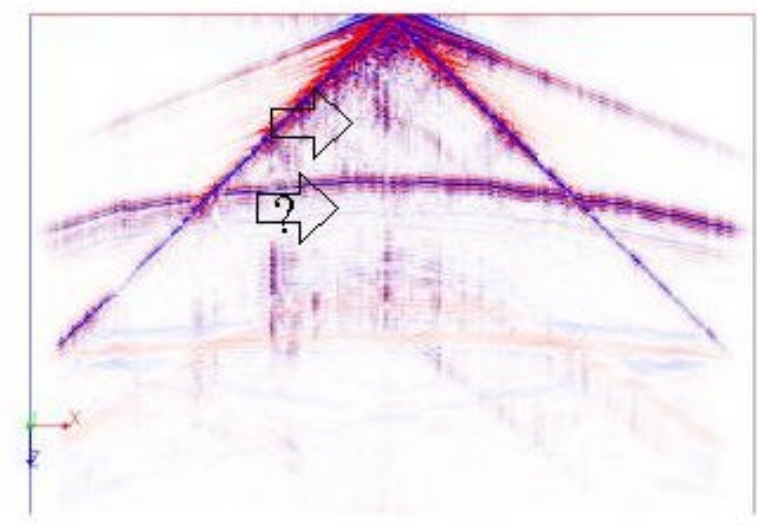

(b) RY64

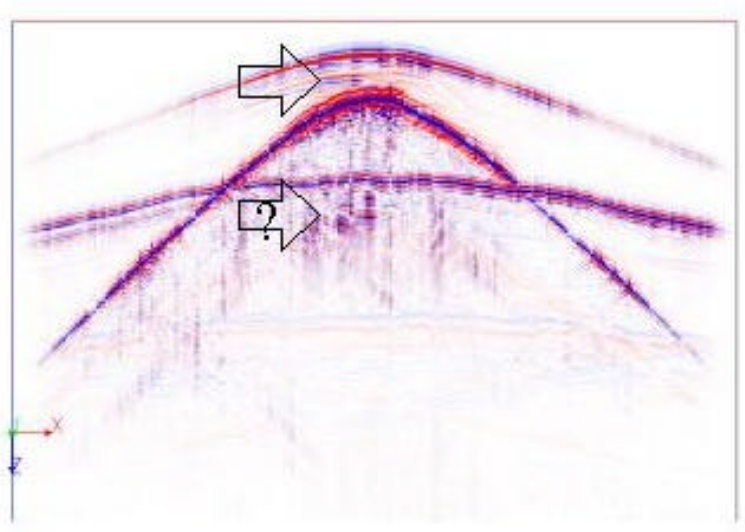

(c) RY37

Figure 4: (a) (c) show shot records observed on RX128, RY64, and RY37. These cross sections are shown in Figure 1. Two kinds of topography-related waves are observed.

\section{Interpretation}

In the series in Figure 3, not only reflected wave from the horizontal interface but also some waves that propagate along the topography are observed. These topography-related waves are distinguished into two types. The first is diffraction energy from a topographic kink point. This could be observed in shot records as strong sideswipe on the lines along the mountain (Figure 4 (b) and (c)). The second is secondary reflection energy from earth's surface. It will be shown as a buried focus on the line across mountain (Figure 4(a)). They are undesirable for our 2D survey. If there is a strong reflector just above a target horizon, the secondary reflection energy may be a serious problem. However, the records in Figure 4 tell the energy is much weaker than the diffraction energy. We should be highly cautious about diffraction from topographic kink point.

RY64 is at a distance from the diffraction source, so it may have better quality compared to 
RY37. However, only a survey along RY64 can be conducted with dense sources and receivers; therefore, the line can be highly acceptable.

\section{Conclusions}

The simulation result suggests that keeping the survey line away from topographic kink points is more important. This result and feasibility of data acquisition, the seismic survey along a mountain ridge will be accepted. In addition, the acoustic wave simulator with a realistic topography model can be useful for our survey planning even though there are several limitations on the method.

\section{Acknowledgements}

This study is one part of the joint research project "Advanced Seismic Imaging Technology phase II" conducted by Japan National Corporation (JNOC), Japan Petroleum Exploration Co., Ltd (JAPEX) and JGI Inc. We all appreciate the contributions to our research from the Exploration Geophysics Laboratory of KYOTO Univ.

\section{Reference}

Takehiko Sato, Toshifumi Matsuoka and Tatsuo Saeki, Three-dimensional wavefield modeling using the Pseudospectral method, Proceeding of the 96th SEGJ conference, 1997, p126-128 